\title{
Simultaneous Overexpression of Integrated Genes by Copy Number Amplification of a Mini-Yeast Artificial Chromosome
}

\author{
Heo-Myung Jung ${ }^{1}$ and Yeon-Hee Kim ${ }^{1,2 *}$ \\ ${ }^{1}$ Department of Smart Bio-Health, Dong-Eui University, Busan 47340, Republic of Korea \\ ${ }^{2}$ Biomedical Engineering and Biotechnology Major, Divison of Applied Bioengineering, Dong-Eui University, Busan 47340, Republic of Korea
}

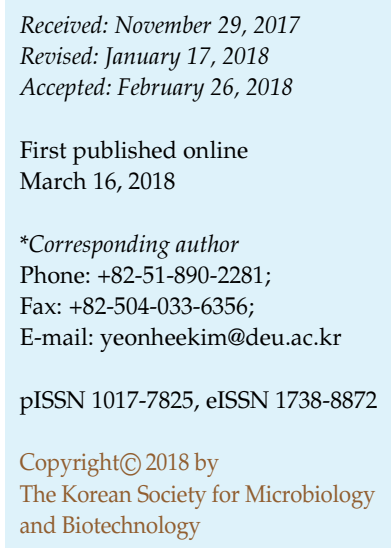

A copy number amplification system for yeast artificial chromosomes (YACs) was combined with simultaneous overexpression of genes integrated into a YAC. The chromosome VII $(1,105 \mathrm{~kb})$ was successfully split to $887 \mathrm{~kb}, 44 \mathrm{~kb}$ containing the element for copy number amplification, and a 184-kb split-YAC. The 44-kb split-mini YAC was amplified a maximum of 9-fold, and the activity of the reporter enzymes integrated into the split-mini YAC increased about 5-7-fold. These results demonstrate that the mini-YAC containing a targeted chromosome region can be readily amplified, and the specific genes in the mini-YAC could be overexpressed by increasing the copy number.

Keywords: Yeast artificial chromosome, PCR-mediated chromosome splitting, copy number amplification, simultaneous overexpression
The yeast artificial chromosome (YAC) system, which allows isolation of larger DNA fragments and easier modification of the cloned DNA than other systems, is an important tool for functional analysis and gene identification [1]. To manipulate large DNA fragments cloned into YACs, various YAC splitting methods have been developed [2-4]. Although YACs have versatile applications, they are limited as single-copy artificial chromosomes. Smith et al. [5] reported the incorporation of elements that enable the amplification of YAC copy number, such as a conditional centromere or the thymidine kinase (TK) gene. If an effective amplification system for YACs is available, the physical mapping and functional analysis of the genome will be simplified, resulting in the ability to increase the copy number of various genes on the YAC. In a previous study, we developed an effective YAC splitting method by incorporating the PCR-mediated chromosome splitting method [6, 7] and reported that the artificial chromosome containing the targeted region of a plant chromosome can be readily amplified [8]. Therefore, we attempted the overproduction of recombinant enzymes and an increase in the expression level of genes in the YAC by introducing the amplification system into a mini-YAC.

In the present study, several enzymes requiring xylan/ xylose metabolism were used as model enzymes, and a mini-YAC harboring a four-gene expression cassette was constructed. The XYLP, XYLB, GRE3, and XYL2 genes encoding endoxylanase, $\beta$-xylosidase, xylose reductase, and xylitol dehydrogenase, respectively, were stably integrated into the yeast chromosome VII in a previous study (unpublished). Saccharomyces cerevisiae SEY2102 $\Delta$ trp / pRS-XylP, pRS-XylB, pRS-Gre3, pRS-Xyl2 strain (designated PBG2 strain), constructed by introducing the integrative plasmid with each gene (pRS-GENE), was used as the host strain for chromosome manipulation.

The YPD nutrient medium and synthetic complete medium for S. cerevisiae and cultivation methods have been described in a previous report [6], and YPDG (YPD containing $1 \%$ galactose) medium was used for galactose-inducible gene expression. To amplify the split-mini YAC, the amplification medium contained $0.67 \%(\mathrm{w} / \mathrm{v})$ yeast nitrogen base; $1 \%(\mathrm{w} / \mathrm{v})$ casamino acids; $3 \%, 4 \%$, and $5 \%(\mathrm{w} / \mathrm{v})$ galactose; $0.8 \mathrm{mg} / \mathrm{ml}$ thymidine; $3 \mathrm{mg} / \mathrm{ml}$ sulfanilamide; and $50 \mu \mathrm{g} / \mathrm{ml}$ methotrexate (S3/M50). Yeast transformation was performed according to the high efficiency transformation protocol [6]. To generate each splitting fragment, pSKcLEU2 [6], pSKURA3, pSKCEN4, and pBGT were used as template plasmids for PCR. The pBGT plasmid, which 
Table 1. Plasmids and primers used in this study.

\begin{tabular}{|c|c|c|}
\hline Plasmids & Description & Products \\
\hline pSKcLEU2 & pBluescript II SK-loxP-CgLEU2 (Candida glabrata LEU2 gene)-loxP gene & SF-I \\
\hline pSKCEN4 & pBluescript II SK-CEN4 gene & SF-II \\
\hline pSKURA3 & pBluescript II SK-URA3 gene & SF-III \\
\hline pBGT & pBluescript II SK-GAL1p/CEN4-TK gene & SF-IV \\
\hline Primers & Sequences $\left(5^{\prime}-3^{\prime}\right)$ & Products \\
\hline ADE3-1 & CTCTATCGGTGCCTCTTCTG & SF-I \\
\hline ADE3-2 & TAGTGAGGGTTAATTGCGCGCTTGGCGTAATCGATGACGGCCTTG & \\
\hline ADE3-3 & TAGTGAGGGTTAATTGCGCGCTTGGCGTAAAGCTTCCAACCAACC & SF-II \\
\hline ADE3-4 & TCCCAATAGTGTTCGTATTA & \\
\hline PDX1-1 & AAGGTGACAAGGTCCTCGAA & SF-III \\
\hline PDX1-2 & TAGTGAGGGTTAATTGCGCGCTTGGCGTAATAGTACTGAAGCAAC & \\
\hline PDX1-3 & TAGTGAGGGTTAATTGCGCGCTTGGCGTAATCTGCGGATGGCTTC & SF-IV \\
\hline PDX1-4 & GTGAGCGACCAGCAACGAGA & \\
\hline SK-F & TTACGCCAAGCGCGCAATTA & All \\
\hline $\operatorname{Tr}-\mathrm{R}$ & ССССААССССААССССААССССААССССАAССССАAТАAТАСGАСТСАСТАТAGGG & \\
\hline
\end{tabular}

Underlined letters indicate overlap sequences used for the second PCR.

PCR products (splitting fragments I-IV) were amplified by PCR using these plasmids as a template and each primer set.

has components for amplifying a split-mini YAC, was constructed by inserting the TK gene and GAL1p/CEN4 into the pBluescript II SK+ vector. The plasmids and oligonucleotides used are listed in Table 1. The pulsed field gel electrophoresis (PFGE) and Southern hybridization carried out have previously been described by Kim et al. [6]. The enzyme activities of endoxylanase, $\beta$-xylosidase, xylose reductase, and xylitol dehydrogenase were measured

A

Chromosome VII (881000 920000 bp)

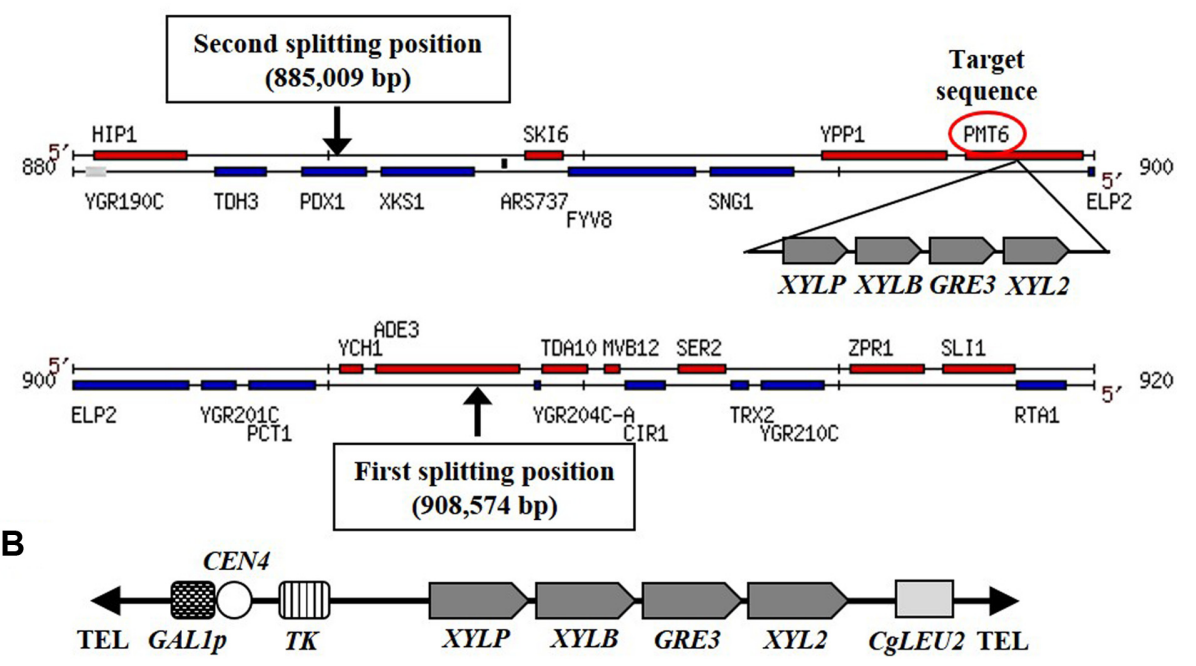

Artificial mini-chromosome (44 kb)

Fig. 1. Splitting position in chromosome VII of Saccharomyces cerevisiae strain PBG2 (A) and structure of the mini-artificial chromosome harboring the xylan metabolism system and copy number amplification system (B).

TEL: Tetrahymena telomeric $\left(\mathrm{C}_{4} \mathrm{~A}_{2}\right)_{6}$ repeat sequence; CEN4: centromere of chromosome IV; GAL1p: promoter of GAL1 gene; TK: thymidine kinase gene; CgLEU2: LEU2 gene of Candida glabrata. 
by each assay method [9-11].

Chromosome VII of the PBG2 strain was selected to split and manipulate because it has the XKS1 gene encoding xylulokinase, and the xylan metabolism-related gene cluster was integrated into the PMT6 gene position (Fig. 1A). In order to manipulate the mini-YAC from chromosome VII, two target genes (ADE3 and PDX1) that do not affect cell viability or growth even when the gene is disrupted or overexpressed by splitting or copy number increase, respectively, were selected for splitting. To split the 184-kb region from the right end of chromosome VII, two splitting fragments (SF-I and SF-II) harboring each target sequence for homologous recombination were amplified. SF-I consisted of $\mathrm{CgLEU2}$, a telomeric $\left(5^{\prime}-\mathrm{C}_{4} \mathrm{~A}_{2}-3^{\prime}\right)_{6}$ repeat sequence, and a target sequence. The $C g L E U 2$ gene used to select yeast transformants was first amplified by PCR using pSKcLEU2 as a template and SK-F and Tr-R as the forward and reverse primers, respectively. Independently, a 500-bp target sequence that corresponded to a sequence from nucleotide positions 908,074 to 908,574 of chromosome VII was amplified by PCR using genomic DNA from the PBG2 strain as template and ADE3-1 and ADE3-2 as primers. A 30-bp overlap sequence was attached to the end of the ADE3-2 primer. The CgLEU2 and target sequence were then used as templates and Tr-R and ADE3-1 as primers for a second PCR to generate the 2.3-kb SF-I. The other SF-II $(1.5 \mathrm{~kb})$ containing CEN4, a telomeric repeat sequence, and the target sequence (nucleotide position 908,575 to 909,075 of Chr. VII) was also amplified by two rounds of PCR using the same procedure as that for SF-I preparation, except with different template DNA and primers. The two amplified splitting fragments were purified and transformed into the PBG2 strain. Four $\mathrm{Leu}^{+}$transformants were analyzed for their karyotypes by PFGE, and three transformants exhibited the expected split-YACs, $925 \mathrm{~kb}$ and $184 \mathrm{~kb}$ from manipulated chromosome VII (1,105 kb) (Fig. 2A). We selected one transformant among the strains with splitYACs and named it the R-split strain. Subsequently, we
A

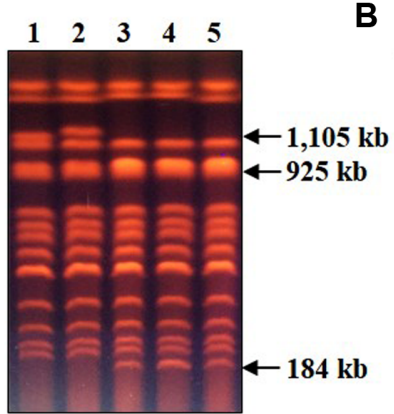

B

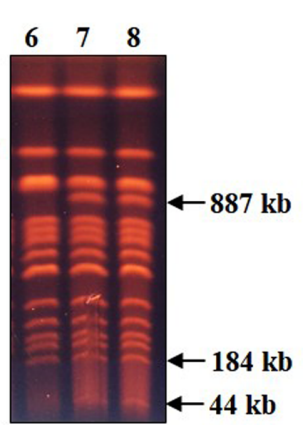

C

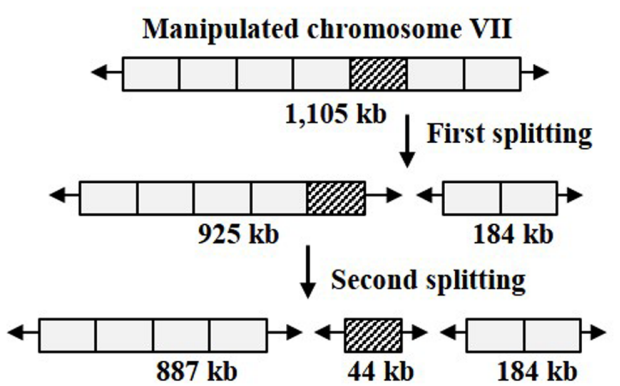

D

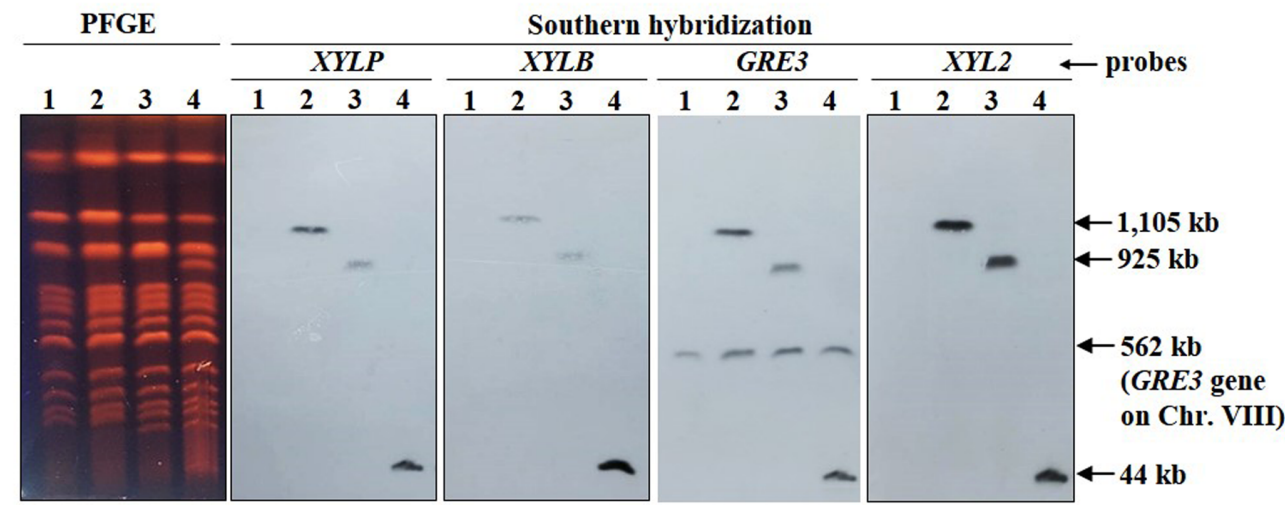

Fig. 2. Analysis of split chromosomes by PFGE (A and B) and schematic diagram of the splitting procedures in chromosome VII by the PCR-mediated chromosome splitting method $(\mathbf{C})$.

Manipulated chromosome VII of 1,105 kb in strain PBG2 was split to 925-kb and 184-kb split YACs (A). Subsequently, the 925-kb split-YAC was more split to $887 \mathrm{~kb}$ and a 44-kb mini-chromosome harboring the xylan degradation system (B). Lane 1: 2102 $\Delta$ trp strain (Host strain); lanes 2-5: Rsplit strain No. 1-4; lanes 6-8: RL-split strain No. 1-3. Confirmation of integration position by PFGE and Southern hybridization (D). XYLP, $X Y L B, G R E 3$, and XYL2 genes were used as probes. Lane 1: 2102Atrp strain (Host strain); lane 2: PBG2 strain; lane 3: R-split strain; lane 4: RL-split strain. 
split the $887-\mathrm{kb}$ region from the left end of chromosome VII. Two splitting fragments (SF-III and SF-IV) harboring each target sequence were amplified using the same procedure used for SF-I and SF-II construction. Briefly, SF-III $(2.2 \mathrm{~kb})$ consisted of the URA3 gene as a selection marker for yeast transformants, a telomeric sequence, and a target sequence (nucleotide positions 884,509 to 885,009 of Chr. VII). Another SF-IV (4 kb) consisted of GAL1P/ CEN4 (GC4), the TK gene, a telomeric sequence, and a target sequence (nucleotide position 885,010 to 885,510 of Chr. VII). To simultaneously introduce an amplification system with chromosome splitting, SF-III and SF-IV were transformed into the R-split strain. Two Ura ${ }^{+}$transformants showed that the 925-kb split-YAC was successfully split into a new 887-kb split-YAC and a 44-kb split-mini YAC containing the amplification system (Figs. 2B and 2C). One transformant was called the RL-split strain. To prove that these new YACs were generated from the splitting of chromosome VII, Southern hybridization was performed using XYLP, XYLB, GRE3, and XYL2 genes as probes, and the probes hybridized to the $44-\mathrm{kb}$ split-mini YAC (Fig. 2D). These observations indicate that the $44-\mathrm{kb}$ fragment

A
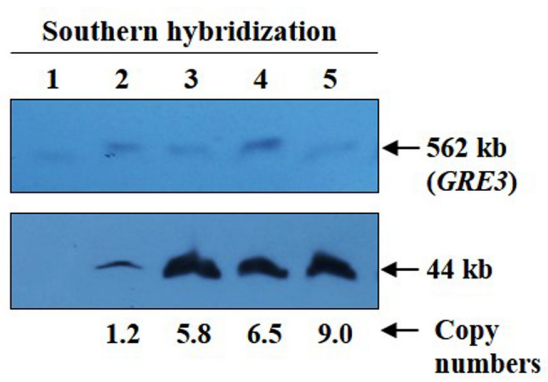

B

\begin{tabular}{cccccc}
\hline Strains & $\begin{array}{c}\text { Cell Growth } \\
\left(\text { O.D }_{600}\right)\end{array}$ & $\begin{array}{c}\text { Endo- } \\
\text { xylanase }\end{array}$ & $\begin{array}{c}\beta \text {-xylo- } \\
\text { sidase }\end{array}$ & XR $^{*}$ & XDH $^{*}$ \\
\hline RL-split & 18.02 & 3.52 & 0.11 & 0.16 & 0.013 \\
CA & 4.72 & 24.72 & 0.57 & 0.91 & 0.085
\end{tabular}

* XR, xylose reductase; XDH, xylitol dehydrogenase

Fig. 3. Confirmation of the copy number amplification by PFGE and Southern hybridization.

(A) GRE3 gene used as probe. The copy numbers of the split-mini YAC were estimated as the ratio of GRE3 on split-mini YAC relative to a single-copy gene, GRE3, on natural chromosome VIII. The intensity of hybridization signals with the GRE3 gene probe was measured using the scion image program. Lane 1: PBG2 strain; lane 2: RL-split strain; lanes 3-5: RL-split strain induced into amplification medium containing $3 \%, 4 \%$ and $5 \%$ galactose, respectively. (B) Comparison of cell growth $\left(\mathrm{OD}_{600}\right)$ and enzyme activity (unit/ml) in RL-split and copy number amplification (CA) strains. harboring the amplification system and the xylan metabolism system originated from the manipulated chromosome VII (Fig. 1B).

To confirm amplification of the copy number of the $44-\mathrm{kb}$ split-mini YAC containing the amplification system, the RL-strain was grown with selective reagents and galactose to evaluate the YAC copy number. The principle of copy number amplification has previously been described [8]. After preparation of DNA plugs, PFGE and Southern hybridization were performed. In YPD medium, the 44-kb split-mini YAC was present at about 1.2 copies per haploid genome (Fig. 3A, lane 2). However, in amplification medium with selective reagent (S3/M50) and $3 \%, 4 \%$, and $5 \%$ galactose, increases in the copy number were observed (Fig. 3A, lanes 3, 4, and 5). The 44-kb split-mini YAC was amplified readily to about 9.0 copies/cell with $5 \%$ galactose. Moreover, endoxylanase activity increased at $24.72 \mathrm{unit} / \mathrm{ml}$ in the copy number amplification strain (CA strain), although cell growth was decreased by adding the selective reagent and galactose, which induce missegregation and slow cell division (Fig. 3B). The activity of $\beta$-xylosidase, xylose reductase, and xylitol dehydrogenase was also successfully increased about 5-7-fold compared with no induction of amplification. These results demonstrate that the mini-YAC containing the targeted chromosome region can be readily amplified, and the specific genes in the miniYAC could be overexpressed by increasing the copy number. This is the first report of simultaneous overexpression of reporter genes by amplifying the copy number of split-mini YAC. This system will provide not only simultaneous overproduction of several enzymes, but also novel strain breeding by allowing transplantation of mini-YACs into industrial strains.

\section{Acknowledgments}

This research was supported by the Basic Science Research Program through the National Research Foundation of Korea (NRF) funded by the Ministry of Science, ICT \& Future Planning (2014R1A1A1003519).

\section{Conflict of Interest}

The authors have no financial conflicts of interest to declare.

\section{References}

1. Burke D, Carle GF, Olson MV. 1987. Cloning of large 
segments of exogenous DNA into yeast by means of artificial chromosome vector. Science 236: 806-811.

2. Pavan WJ, Hieter P, Sears D, Burkhoff A, Reeves RH. 1991. High-efficiency yeast artificial chromosome fragmentation vectors. Gene 106: 125-127.

3. Emanuel SL, Cook JR, O'Rear J, Rothstein R, Pestka S. 1995. New vectors for manipulation and selection of functional yeast artificial chromosomes (YACs) containing human DNA inserts. Gene 155: 167-174.

4. Kim YH, Kaneko Y, Fukui K, Kobayashi A, Harashima S. 2005. A yeast artificial chromosome-splitting vector designed for precise manipulation of specific plant chromosome region. J. Biosci. Bioeng. 99: 55-60.

5. Smith DR, Smyth AP, Moir DT. 1990. Amplification of large artificial chromosomes. Proc. Natl. Acad. Sci. USA 87: 82428246.

6. Kim YH, Sugiyama M, Yamagishi K, Kaneko Y, Fukui K, Kobayashi A, et al. 2005. A versatile and general splitting technology for generating targeted YAC subclones. Appl. Microbiol. Biotechnol. 69: 65-70.
7. Sugiyama M, Ikushima S, Nakazawa $T$, Kaneko $\mathrm{Y}$ Harashima S. 2005. PCR-mediated repeated chromosome splitting in Saccharomyces cerevisiae. Biotechniques 38: 909-914.

8. Kim YH, Nam SW. 2010. Development of simultaneous YAC manipulation-amplification (SYMA) system by chromosome splitting technique harboring copy number amplification system. J. Life Sci. 20: 789-793.

9. Chun YC, Jung KH, Lee JC, Park SH, Chung HK, Yoon KH. 1998. Molecular cloning and the nucleotide sequence of a Bacillus sp. KK-1 $\beta$-xylosidase gene. J. Microbiol. Biotechnol. 8: 28-33.

10. Lee LH, Kim DY, Han MK, Oh HW, Ham SJ, Park DS, et al. 2009. Characterization of an extracellular xylanase from Bacillus sp. HY-20, a bacterium in the gut of Apis mellifera. Korean J. Microbiol. 45: 332-338.

11. Kim SR, Kwee NR, Kim B, Jin YS. 2013. Feasibility of xylose fermentation by engineered Saccharomyces cerevisiae overexpressing endogenous aldose reductase (GRE3), xylito dehydrogenase (XYL2), and xylulose kinase (XYL3) from Scheffersomyces stipitis. FEMS Yeast Res. 13: 312-321. 